\title{
Vector Constants of Motion for Time-Dependent Kepler and Isotropic Harmonic Oscillator Potentials
}

\author{
O.M. Ritter, \\ Departamento de Física, \\ Universidade Federal de Santa Catarina, Trindade \\ 88040-900 Florianópolis SC, Brasil \\ F.C. Santos, and A. C. Tort ${ }^{\ddagger}$ \\ Instituto de Física, Universidade Federal do Rio de Janeiro \\ Cidade Universitária, Caixa Postal 68528 \\ 21945-970 Rio de Janeiro RJ, Brasil
}

Received on 8 June, 2000. Revised version received on 21 March, 2001.

We discuss a method for obtaining vector constants of motion for time-dependent central fields.

\section{Introduction}

The search for first integrals has assumed an increasing importance in the determination of the integrability of a dynamical system. The notion of integrability is related to the existence of first integrals of motion. Several methods of finding first integrals are available in the literature. Some of them are based on the symmetries of the system [1], [2]. Another possibility relies on a direct search for invariants with a specified form (usually, a polynomial form on the velocities) [3], [4]. Still other methods to determine the integrability of a system are available as, for instance, the Painlevé Analysis [5]. One of the most aesthetically appealing and important problem in physics is the central field problem [6]. Energy and angular momentum associated with this type of field are well known conserved quantities. However, other vector and tensor conserved quantities have been associated with some particular central fields. The Laplace-Runge-Lenz vector [7] is a vector first integral of motion for the Kepler problem; the Fradkin tensor [8] is conserved for the case of the harmonic oscillator and for any central field it is possible to find a vector first integral of motion as was shown in [9]. In the general case these additional integrals of motion turn out to be complicated functions of the position $\mathbf{r}$ and linear momentum $\mathbf{p}$ of the particle probing the central field. When orbits are closed and degener-

\footnotetext{
e-mail: fsc1omr@fisica.ufsc.br

$\dagger$ e-mail: filadelf@if.ufrj.br

‡e-mail:tort@if.ufrj.br
}

ated with respect to the mechanical energy, however, we should expect these additional constants of motion to be simple functions of $\mathbf{r}$ and $\mathbf{p}$. In this article we wish to exploit further this line of reasoning by determining the existence of such additional vector first integrals of motion for some special class of time-dependent Kepler and isotropic harmonic oscillator problems. In particular, we will show that for the time-dependent Kepler problem the existence of a vector constant of motion coupled to a simple transformation of variables turns the problem easily integrable.

\section{Constructing vector con- stants of motion}

The force $\mathbf{f}(\mathbf{r}, t)$ acting on a particle moving in a central but otherwise arbitrary and possible time-dependent field of force $g(r, t)$ can be written as

$$
\mathbf{f}(\mathbf{r}, t)=g(r, t) \mathbf{r},
$$

where $\mathbf{r}=\mathbf{r}(t)$ is the position vector with respect to the center of force, $r$ is its magnitude, and $t$ is the time. Assuming a constant vector $\mathbf{j}$ of the form

$$
\mathbf{j}(\mathbf{p}, \mathbf{r}, t)=A(\mathbf{p}, \mathbf{r}, t) \mathbf{p}+B(\mathbf{p}, \mathbf{r}, t) \mathbf{r},
$$


where $\mathbf{p}=\mathbf{p}(t)=m \dot{\mathbf{r}}(t)$ is the linear momentum, $m$ is the reduced mass and $A, B$ are arbitrary scalar functions of $\mathbf{p}, \mathbf{r}$ and $t$, it follows that the functions $A$ and $B$ must satisfy

$$
\begin{aligned}
& A g+\frac{d B}{d t}=0 \\
& \frac{d A}{d t}+\frac{B}{m}=0 .
\end{aligned}
$$

Eliminating $B$ between (3) and (4) we obtain

$$
m \frac{d^{2} A}{d t^{2}}-g A=0
$$

It follows from (4) that $\mathbf{j}$ can be written in the form

$$
\mathbf{j}=A \mathbf{p}-m \frac{d A}{d t} \mathbf{r} .
$$

If the field $g(r, t)$ is known any solution of (5) will yield a vector constant of motion of the form given by (6). Equation (5), however, is a differential equation whose solution may turn out to be a hard task to accomplish. Nevertheless, we can make progress if instead of trying to tackle it directly we make plausible guesses concerning $A$ thereby linking $\mathbf{j}$ to specific forms of the field $g(r, t)$.

\section{Time-dependent fields}

Let us consider time-dependent central force fields for which we can build more general vector first integrals of motion. As with the time-independent case there are of course several possibilities when it comes to the choice of a function $A$ for a time-dependent central field. Here is one

$$
A=\phi(t) \mathbf{r} \cdot \mathbf{p}+\psi(t) \mathbf{r} \cdot \mathbf{r} .
$$

Using (7) in (5) and assuming the additional condition

$$
\psi+m \frac{d \phi}{d t}=0
$$

we arrive at

$$
g(r, t)=\frac{m}{\phi} \frac{d^{2} \phi}{d t^{2}}+\frac{C}{\phi r^{3}} .
$$

The vector first integral of motion associated with (9) is

$$
\mathbf{j}=m \phi^{2} \mathbf{L} \times \frac{d}{d t}\left(\frac{\mathbf{r}}{\phi}\right)-\frac{m C \mathbf{r}}{r}
$$

where we have made use of the fact that the angular momentum is constant for any arbitrary central field whether it is time-independent or not. If in (10) we set $\phi=1$ and $C \neq 0$, we can recover the time-independent Kepler problem. If we set $(m / \phi) d^{2} \phi / d t^{2}=-k(t)$, that is, if $\phi$ is an arbitrary function of the time, and also $C=0$, we have the time-dependent isotropic harmonic oscillator field, $\mathbf{F}(r)=-k(t) \mathbf{r}$. In this case $\mathbf{j}$ is equal to the first term on the R.H.S. of (10). If $(m / \phi) d^{2} \phi / d t^{2}=-k$ and $C=0$, we have the timeindependent isotropic harmonic oscillator field. Finally, if $(m / \phi) d^{2} \phi / d t^{2}=0, \phi \neq 0$ for any instant of time $t$ and $C \neq 0$, we have a time-dependent Kepler field. In this case

$$
g(r, t)=\frac{C}{\left(c_{1} t+c_{0}\right) r^{3}},
$$

and the associated constant vector is a time-dependent generalization of the Laplace-Runge-Lenz vector

$$
\mathbf{j}=m\left(c_{1} t+c_{0}\right)^{2} \mathbf{L} \times\left(\frac{d \mathbf{r} / d t}{c_{1} t+c_{0}}-\frac{c_{1} \mathbf{r}}{\left(c_{1} t+c_{0}\right)^{2}}\right)-\frac{m C \mathbf{r}}{r}
$$

As another example let us consider the timedependent isotropic harmonic oscillator and show how it is possible to generalize the Fradkin tensor for this case. Let the function $A(\mathbf{r}, \mathbf{p}, t)$ be written as

$$
A=\phi(t) \hat{\mathbf{u}} \cdot \mathbf{r}+\psi(t) \hat{\mathbf{u}} \cdot \mathbf{p} .
$$

Using (5) and assuming the additional condition

$$
2 \frac{d \phi}{d t}+m \frac{d^{2} \psi}{d t^{2}}=0
$$

we obtain

$$
m \frac{d^{3} \psi}{d t^{3}}-4 g \frac{d \psi}{d t}-2 \frac{d g}{d t} \psi=0
$$

We can solve (15) thoroughly if $g(r, t)$ is a function of the time $t$ only. In this case, as before, we end up with the time-dependent isotropic harmonic oscillator. From (13) and (14) we can derive the vector $\mathbf{j}$ associated with (13) which, in terms of components reads

$$
j_{i}=F_{i j} u_{j}
$$

where $F_{i j}$ is a generalized Fradkin tensor and can be written as 


$$
F_{i j}=\left(-\frac{m}{2} \frac{d \psi}{d t}\right) p_{i} x_{j}+\psi p_{i} p_{j}+\left(\frac{m^{2}}{2} \frac{d^{2} \psi}{d t^{2}}-m g \psi\right) x_{i} x_{j}-\frac{m}{2} \frac{d \psi}{d t} x_{i} p_{j}
$$

It is not hard to see that the trace of this generalized Fradkin tensor $\mathbf{F}(\mathbf{r}, \mathbf{p}, t)$ becomes the energy of the particle when $g(r, t)$ is a constant field. isotropic harmonic oscillator potentials. But firstly we must establish some very general relationships. First notice that (6) can be recasted into the form

$$
\mathbf{j}=m A(\mathbf{r}, \mathbf{p}, t)^{2} \frac{d}{d t}\left[\frac{\mathbf{r}}{A(\mathbf{r}, \mathbf{p}, t)}\right] .
$$

Now we will show how to take advantage of the vector constant $\mathbf{j}$ to obtain the solution for the Kepler and the
Integrating (18) we readily obtain

$$
\frac{\mathbf{r}(t)}{A(\mathbf{r}, \mathbf{p}, t)}-\frac{\mathbf{r}(0)}{A(\mathbf{r}(0), \mathbf{p}(0), 0)}=\frac{\mathbf{j}}{m} \int_{0}^{t} \frac{d \tau}{A(\mathbf{r}(\tau), \mathbf{p}(\tau), \tau)^{2}}
$$

Let us now show how we can obtain the orbits in the case of the time-dependent Kepler problem. In what follows, we will be dealing with a special class of timedependent Kepler problems, namely, those satisfying (9). In polar coordinates the angular momentum conservation law is written in the form

$$
l=m r^{2} \frac{d \theta}{d t}
$$

and this allows to rewrite (18) as

$$
\mathbf{j}=l\left[-\frac{d}{d \theta}\left(\frac{A}{r}\right) \hat{\mathbf{r}}+\frac{A}{r} \hat{\theta}\right]
$$

where we have introduced the polar unitary vectors $\hat{\mathbf{r}}$ and $\hat{\theta}$. The component of the vector $\mathbf{j}$ in the direction of $\hat{\theta}$ is given by

$$
\mathbf{j} \cdot \hat{\theta}=l \frac{A}{r} .
$$

In section 3 we determined a generalized LaplaceRunge-Lenz vector for the time-dependent Kepler problem. The scalar function $A(\mathbf{r}, \mathbf{p}, t)$ associated with this vector was found to be

$$
A=\phi(t) \mathbf{r} \cdot \mathbf{p}-m \frac{d \phi}{d t} r^{2}
$$

Considering $A$ as a function of $\theta$ we can write

$$
\frac{A}{r}=-l \frac{d}{d \theta}\left(\frac{\phi}{r}\right) .
$$

Equations (22) and (24) lead to

$$
\frac{d}{d \theta}\left(\frac{\phi}{r}\right)=-\frac{\mathbf{j} \cdot \hat{\theta}}{l^{2}}=\frac{j}{l^{2}} \sin (\theta-\alpha),
$$

where $\alpha$ is the angle between $\mathbf{j}$ and the $\mathcal{O} \mathcal{X}$ axis. Upon integrating (25) we find

$$
\frac{\phi}{r}=\frac{\phi_{0}}{r_{0}}+\frac{j}{l^{2}}\left[\cos \left(\theta_{0}-\alpha\right)-\cos (\theta-\alpha)\right] .
$$

This is the orbit equation we were looking for.

It is possible to transform the time-dependent $\mathrm{Ke}-$ pler problem into the time-independent one. In order to see this we define a new position vector $\mathbf{r}^{\prime}$ according to

$$
\mathbf{r}^{\prime}=\frac{\mathbf{r}}{\phi}
$$

and redefine our time parameter according to

$$
d t^{\prime}=\frac{d t}{\phi^{2}}
$$


We can recast the equation of motion for the timedependent Kepler problem, namely

$$
m \frac{d^{2} \mathbf{r}}{d t^{2}}=\left(\frac{m}{\phi} \frac{d^{2} \phi}{d t^{2}}+\frac{C}{\phi r^{3}}\right) \mathbf{r}
$$

into a simpler form. Making use of the above transformations the equation of motion (29) can be written as

$$
m \frac{d^{2} \mathbf{r}^{\prime}}{d t^{\prime 2}}=\frac{C}{\left(r^{\prime}\right)^{3}} \mathbf{r}^{\prime} .
$$

Equation (30) corresponds to the usual timeindependent Kepler problem. Equations (27) and (28) show that the open solutions of (30) are transformed into the open solutions of (29) with the same angular size and that closed solutions of (30) are associated with spiraling solutions of (29). To conclude consider the total mechanical energy associated with (30)

$$
E=\frac{\mathbf{p}^{\prime 2}}{2 m}+\frac{C}{r^{\prime}}=\text { const. }
$$

Since $\mathbf{p}^{\prime}$ and $\mathbf{p}$ are related by

$$
\mathbf{p}^{\prime}=\phi(t) \mathbf{p}-m \dot{\phi}(t) \mathbf{r}
$$

and $r^{\prime}$ and $r$ by (27) we easily obtain

$$
E=\phi^{2} \frac{\mathbf{p}^{2}}{2 m}-2 \phi \frac{d \phi}{d t} \mathbf{r} \cdot \mathbf{p}+\left(\frac{d \phi}{d t}\right)^{2} \frac{r^{2}}{2}+C \frac{\phi}{r}
$$

which is a conserved quantity and can be interpreted as a generalization of the energy of the particle under the action of a time-dependent Kepler field.

\section{Conclusions}

In this paper we have outlined a simple and effective method for treating problems related with timedependent and time independent central force fields. In particular we have dealt with the Kepler problem and the isotropic harmonic oscillator fields. The difficulty in finding vector constants of motion for central fields stems from the fact that, in general, orbits for this type of problem are not closed, therefore any new ways to attack those problems are welcome. In our method this difficulty is transferred, so to speak, to the obtention for each possible central field, which can be time-dependent or not, of a certain scalar function of the position, linear momentum and time. For a given central field this scalar function is a solution of (5). In the general case, the obtention of the scalar function is a difficult task. Judicious guesses, however, facilitate the search for solution of (5) and this is what we have done here. Notice also that the method apllies to closed orbits $\phi_{0}<1$ as well as open orbits $\phi_{0}>1$. The case $\phi_{0}=1$, the parabolic orbit is also contemplated.

\section{References}

[1] P.J. Olver, 1986 Applications of Lie groups to differential equations (New York: Springer Verlag).

[2] F. Cantrijn and W. Sarlet, Siam Review 23467 (1981).

[3] E.T. Whittaker, A Treatise on the Analytical Dynamics of Particles and Rigid Bodies $4^{\text {th }}$ ed. (Cambridge: Cambridge University Press), 1937.

[4] J. Hietarinta, Physics Reports 147, 87 (1987).

[5] M.J. Ablowitz, A. Ramani, and H. Segur Lett. Nuovo Cimento 23, 333 (1978).

[6] H. Goldstein Classical Mechanics $2^{\text {nd }}$ ed (Reading MA: Addison-Wesley), 1980.

[7] Laplace P S 1799 Traité de Mécanique Celeste; Runge C Vektoranalysis V1 pg. 70 (Leipzig: S. Hirzel, Leipzig), 1919; W Pauli Z. Phyisik 36, 336-63 (1926); Lenz W Z. Phyisik 24, 197 (1926); Heintz W H Am. J. Phys. 42, 1078 (1974). See also Goldstein H Am. J. Phys. 43, 737 (1975); ibid. 44, 1123 (1976).

[8] Fradkin D M Am. J. Phys. 33, 207 (1965); Prog. Theor. Phys. 37, 798 (1967).

[9] C.C. Yan, J. Phys. A 24, 4731 (1991). 